\title{
To Drown A Baby in A Water Bottle. Case of A Severe Hyponatremia Caused by Fluid Oversupply in A 2-Year-Old Child
}

ISSN: 2576-9200

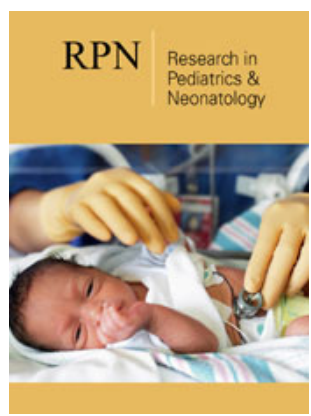

*Corresponding author: Agnieszka Zubkiewicz-Kucharska, Department of Pediatric Endocrinology and Diabetology, Wrocław Medical University, Poland

Submission: 眇 December08, 2020

Published: 湝January 25, 2021

Volume 5 - Issue 2

How to cite this article: Agnieszka Zubkiewicz-Kucharska. To Drown A Baby in A Water Bottle. Case of A Severe Hyponatremia Caused by Fluid Oversupply in A 2-Year-Old Child. Research in Pediatrics \& Neonatology. 5(2). RPN. 000609. 2021.

DOI: $10.31031 /$ RPN.2021.05.000609

Copyright $\subset$ Agnieszka ZubkiewiczKucharska. This article is distributed under the terms of the Creative Commons Attribution 4.0 International License, which permits unrestricted use and redistribution provided that the original author and source are credited.
Agnieszka Zubkiewicz-Kucharska ${ }^{1 *}$

${ }^{1}$ Department of Pediatric Endocrinology and Diabetology, Wrocław Medical University, Poland

Introduction

Sodium concentration in the body is controlled by osmoregulatory system, which regulate water intake and excretion. If this system fails and plasma sodium level stray outside its normal range (135-145mmol/L), the cells are exposed to hypotonic or hypertonic stress. Hyponatremia is one of the most common electrolyte imbalances, occurring in up to $20 \%$ of hospitalized patients. It is defined as a plasma sodium concentration lower than $135 \mathrm{mmol} / \mathrm{L}$, with severe hyponatremia being below $120 \mathrm{mmol} / \mathrm{L}$. The symptoms of hyponatremia vary, depending not only on the concentration of sodium, but also on the rate of lowering of natremia. Mild symptoms may include headaches, cognitive impairment, lethargy, nausea, and vomiting; however, the signs may be subtle, or it may be asymptomatic whatsoever. In severe hyponatremia consciousness disturbances, seizures, and coma are common. The most common cause of hyponatremia include vomiting and diarrhea, adrenal insufficiency, heart, liver and renal failure, syndrome of inappropriate antidiuretic hormone secretion (SIADH), cerebral salt wasting syndrome, osmotic diuresis, and hyperglycemia. One should not forget about overhydration as a potential reason of hyponatremia.

Here we present a case of a child with severe hyponatremia due to fluid oversupply. A twoyear-old boy was admitted to the Emergency Department after an episode of seizures lasting several minutes, with breathing disorders and loss of consciousness. Past medical history was not relevant, the psychomotor and physical development was normal (body mass $14 \mathrm{~kg}=72 \mathrm{pc}$, height $91,5 \mathrm{~cm}=63 \mathrm{pc}$, BMI $16,7 \mathrm{~kg} / \mathrm{m}^{2}=75 \mathrm{pc}$ ). Laboratory examination revealed a decreased natremia of $120 \mathrm{mmol} / \mathrm{L}$, which was gradually corrected with $3 \%$ sodium chloride, with the improvement in the child's general condition. The symptoms have withdrawn without any sequalae. The remaining laboratory tests as well as neuroimaging studies revealed no severe irregularities. Also, the results of the additional tests performed after the normalization of the child's condition did not cause concern. Kidney and liver function were normal. There were no cardiological disorders. Adrenal insufficiency, hypothyroidism and hyperglycemia were all excluded.

In the following days, concentrations of electrolytes as well as osmolarity of serum and urine were within normal ranges. In the first days of treatment, polydipsia (approximately $4 \mathrm{~L}$ /day), and polyuria of 4 liters of urine/day were observed, but the fluid balance was maintained. It was noticed that the boy drank on demand, was taught to drink water at night, and was calmed down with a water bottle. Mother confirmed that the same was happening at home, before the hospitalization. Fluid restriction test showed proper increase in urine osmolarity while serum osmolarity remained stable. Moreover, the dehydration-stimulated copeptin concentrations were adequate to serum osmolarity and indicated psychogenic polydipsia. The age-appropriate fluid supply was recommended of $1.1 \mathrm{~L} / \mathrm{day}$. In the followup, the plasma sodium concentration as well as serum and urine osmolarity and gravity are stabilized in the normal ranges. The child does not present any distressing symptoms.

The detrimental effects of dehydration are well known, however the adverse effects of overhydration remain less described. If there is no malfunction of antidiuretic hormone 
(ADH) feedback loop nor ADH concentration is increased, the hyponatremia may result from failure of the hypothalamic-kidney feedback loop, overwhelmed by increased fluid intake. However, if osmoregulation is not disturbed, both thirst as well as ADH secretion are inhibited when the sodium concentration is decreased below 135mmol/L. Furthermore, it was shown that aquaporin 2 (AQP2) water channels within the kidney collecting duct cells are downregulated as a consequence of a chronic overhydration (lasting more than three days). As a response to fluid oversupply, the ADH level is lowered, followed by an increase of urinary free water excretion in order to adapt to enlarged fluid intake. Moreover, it should be underlined that ADH secretion is stimulated by hypovolemia, so water will be retained even if hyponatremia occurs. It may be especially dangerous in the situation of drinking hypotonic fluids such as apple juice, tea, or spring water-like it was in the presented case [1-3].

The most serious, even fatal, consequence of hyponatremia due to excessive water consumption is cerebral edema. It was estimated that rapid (in less than an hour) intake of approximately 3-4L of fluids may trigger symptomatic hyponatremia in a healthy subject. Obviously, higher volumes (up to 10-20L/day) are tolerated when consumed slower. Drinking above the physiological need to prevent the detrimental effect of dehydration has been popularized nowadays. The most popular recommendation is to drink at least $2 \mathrm{~L}$ of water per day to achieve health benefits, however in many people the fluid intake is even greater. It can only be assumed that by constantly giving her baby water, the mother wanted to protect the boy from dehydration, unfortunately causing water intoxication. As so, it is worth to notice that increased water consumption may not be completely benign.

\section{References}

1. Sterns RH (2015) Disorders of plasma sodium--causes, consequences, and correction. N Engl J Med 372(1): 55-65.

2. Refardt J, Winzeler B, Crain MC (2019) Copeptin and its role in the diagnosis of diabetes insipidus and the syndrome of inappropriate antidiuresis. Clin Endocrinol (Oxf) 91(1): 22-32.

3. Butler TH, Hale VS, McGrandy AP, VanSumeren M (2019) Of mice and men-the physiology, psychology, and pathology of overhydration. Nutrients 11(7): 1539. 